\title{
Chaotic particle dynamics in viscous flows: The three-particle Stokeslet problem
}

\author{
Imre M. Jánosi, ${ }^{1, *}$ Tamás Tél, ${ }^{2}$ Dietrich E. Wolf, ${ }^{1}$ and Jason A. C. Gallas ${ }^{1,3}$ \\ ${ }^{1}$ HLRZ, Forschungszentrum Jülich, D-52425 Jülich, Germany \\ ${ }^{2}$ Institute for Theoretical Physics, Eötvös University, Puskin utca 5-7, H-1088 Budapest, Hungary \\ ${ }^{3}$ Instituto de Física, Universidade Federal do Rio Grande do Sul, 91501-970 Porto Alegre, Brazil
}

(Received 17 December 1996)

\begin{abstract}
It is well known, that the dynamics of small particles moving in a viscous fluid is strongly influenced by the long-range hydrodynamical interaction between them. Motion at high viscosity is usually treated by means of the Stokes equations, which are linear and instantaneous. Nevertheless, the hydrodynamical interaction mediated by the liquid is nonlinear; therefore the dynamics of more than two particles can be rather complex. Here we present a high resolution numerical analysis of the classical three-particle Stokeslet problem in a vertical plane. We show that a chaotic saddle in the phase space is responsible for the extreme sensitivity to initial configurations, which has been mentioned several times in the literature without an explanation. A detailed analysis of the transiently chaotic dynamics and the underlying fractal patterns is given.
\end{abstract}

[S1063-651X(97)10408-1]

PACS number(s): 47.10. $+\mathrm{g}$, 47.15.Gf, 47.52. $+\mathrm{j}, 05.45 .+\mathrm{b}$

\section{INTRODUCTION}

When microscopic objects move in a fluid, the stresses in the liquid due to viscosity may be several orders of magnitude larger than those due to inertia. A first estimate of the ratio

$\frac{\text { (stress in fluid due to inertia) }}{\text { (stress in fluid due to viscosity) }}$

is given by the Reynolds number $\operatorname{Re}$ as $\operatorname{Re}=L U \varrho / \mu$, where $L$ and $U$ denote the characteristic length and speed of the particle, $\varrho$ and $\mu$ are the density and the dynamic viscosity of the fluid. Collective phenomena involving the motion of assemblies of small particles in the low Reynolds number limit cover a wide range of interest to both scientists and engineers. Probably the most extensively studied classical problem is the passive sedimentation of particles in the gravitational field [1]. A closely related system is that of small charged or polarized particles in a viscous fluid driven by an external electrical field, the dynamics of which is often referred to as electrophoretic motion [2]. Similar physics is involved in another, more complicated example for translation in a strongly viscous environment, the swimming of microorganisms such as algae and bacteria. In a quiescent fluid even their slow motion (typically a couple of meters per day) can result in considerable spatial rearrangements, dynamic pattern formation, or bioconvection [3] due to hydrodynamical interactions.

The familiar approximation, which gives reasonably accurate results up to $\operatorname{Re} \approx 1$, is based on the Stokes equations [1]

$$
\begin{gathered}
\boldsymbol{\nabla} \cdot \mathbf{u}=\mathbf{0}, \\
-\nabla p+\mu \Delta \mathbf{u}+\mathbf{f}=\mathbf{0} .
\end{gathered}
$$

\footnotetext{
*Permanent address: Department of Atomic Physics, Eötvös University, Puskin u. 5-7, H-1088 Budapest, Hungary.
}

Here $\mathbf{u}=\mathbf{u}(\mathbf{r})$ is the velocity field, $p=p(\mathbf{r})$ and $\mathbf{f}=\mathbf{f}(\mathbf{r})$ denote the local pressure and force fields, respectively. Equation (1) expresses the incompressibility of the flow, while Eq. (2) is the dynamical equation obtained by neglecting the inertial terms in the full Navier-Stokes equation due to the slow temporal changes characterizing the process. The most important simplification represented by the Stokes equations (1) and (2) is that they are linear and instantaneous [4]. These equations are solved subject to boundary conditions most often on the velocity u. Usually the so called "no slip" condition is prescribed on solid surfaces, which means that the relative velocity between the fluid and the body vanishes on the boundary [1].

In this work we revisit a classical problem of viscous flows, namely, the dynamics of three microscopic, nonBrownian particles driven by constant external forces, like gravity, or electrostatic field. To achieve the highest possible resolution in the initial configuration space with acceptable computational demand, we used the simplest approximation capturing the essence of hydrodynamical interaction, the Stokeslet model, described below. The more complete Stokes, or creeping flow, solutions for a couple of particles can be obtained by a number of special techniques tailored to the viscous limit [1,5-15]. Although these equations of motion are relatively simple, long-range interactions and complicated boundary shapes usually require further simplifying assumptions [16]. Up to now, lattice Boltzmann techniques $[17,18]$ have come closest to realistic simulations of rather large systems at wide Re ranges. In a remarkable recent computation, Ladd [19] treated 32768 suspended particles at low Reynolds numbers, and he could track the trajectories for about 500 Stokes times. (A unit Stokes time is defined as the time needed for an isolated sphere to pass over a distance of one-particle radius.) In our Stokeslet model simulations, we followed particle trajectories for several thousands Stokes times from about a million initial configurations which would have been unfeasible with more sophisticated techniques. 
The paper is organized as follows. In Sec. II we briefly outline the Stokeslet model for $n$ interacting particles. Next (Sec. III) the motion of three particles in a vertical plane is studied in the framework of this model. Results of high resolution numerical simulations are presented in Sec. IV. We show that a chaotic saddle existing in the phase space is responsible for the extreme sensitivity to initial configurations which has been mentioned several times in the literature without an explanation. A detailed analysis of the transiently chaotic dynamics and the underlying fractal patterns is given. Section V is devoted to a discussion of the range of validity of our results.

\section{THE STOKESLET MODEL}

The simplest possible treatment of a viscous two-phase flow is based on the so-called Stokeslet model [1], where the moving objects are approximated by pointlike particles. Hocking [20] first studied the motion of small clusters of such particles by means of this approximation in order to explain related sedimentation experiments by Jayaweera, Mason, and Slack [21]. Recent analytical treatments for a restricted class of initial configurations add to the interest in the Stokeslet approximation [22].

The Stokeslet velocity distribution is the solution to Eq. (2) with a little spherical particle of radius $a$ moving with velocity $\mathbf{v}_{0}$ in an unbounded fluid which is at rest at infinity [16]. A particle momentarily in the origin generates the velocity field

$$
\mathbf{u}(\mathbf{r})=\frac{3 a v_{0}}{4}\left(\frac{\mathbf{e}}{r}+\frac{(\mathbf{e} \cdot \mathbf{r}) \mathbf{r}}{r^{3}}\right)=a v_{0} \mathbf{U}(\mathbf{r}), \quad r>a .
$$

Here $\mathbf{e}$ is the unit vector in the direction of the self-velocity $\mathbf{v}_{\mathbf{0}}=v_{0} \mathbf{e}, r=\|\mathbf{r}\|$, and terms of $O\left(a^{3} / r^{3}\right)$ are neglected. In the case of sedimentation, e.g., $\mathbf{e}$ is the unit vector pointing downward along the vertical $(z)$ axis. In the following, however, we usually refer to e as directed vertically upward in order to emphasize the generality of the treatment, and indicate the relevance to, e.g., electrophoresis, too.

Let us now consider $n$ particles which would all have the same steady state velocity $\mathbf{v}_{0}$ if they were isolated. In the case of sedimentation or electrophoresis, $v_{0}=F \varrho /(6 \pi \mu a)$ due to Stokes law, where $F$ is the modulus of the constant external force. Then, particle $j$ generates a velocity $\mathbf{u}\left(\mathbf{r}-\mathbf{r}_{j}\right)$ at point $\mathbf{r}$. The linearity of Eqs. (1) and (2) implies in the lowest order approximation that we can use free superposition. Thus, the velocity distribution due to $n$ particles is $\sum_{j=1}^{n} \mathbf{u}\left(\mathbf{r}-\mathbf{r}_{j}\right)$. At position $\mathbf{r}_{i}$ of particle $i$ the background flow generated by the other $n-1$ particles is $\sum_{j \neq i}^{n} \mathbf{u}\left(\mathbf{r}_{i}-\mathbf{r}_{j}\right)$. In the lowest order approximation one assumes that the velocity of the particle $\dot{\mathbf{r}}_{i}$ at $\mathbf{r}_{i}$ in the laboratory frame is the sum of this passive advection velocity and $\mathbf{v}_{0}$

$$
\dot{\mathbf{r}}_{i}=\mathbf{v}_{\mathbf{0}}+a v_{0} \sum_{j \neq i}^{n} \mathbf{U}\left(\mathbf{r}_{i}-\mathbf{r}_{j}\right), \quad i=1, \ldots, n .
$$

It is useful to measure the length and the time in units of $a$ and $a / v_{0}$ (Stokes time), respectively. The dimensionless form of Eq. (4) then is

$$
\dot{\mathbf{r}}_{i}=\mathbf{e}+\sum_{j \neq i}^{n} \mathbf{U}\left(\mathbf{r}_{i}-\mathbf{r}_{j}\right), \quad i=1, \ldots, n
$$

With the given expression of $\mathbf{U}$ [see Eq. (3)], this defines a closed set of differential equations for the trajectories $\mathbf{r}_{i}(t)$ of the different particles. Because particles are assumed to be pointlike, vectors $\mathbf{r}_{i}$ and $\mathbf{r}_{j}$ must not coincide but otherwise they can take on any values. With a fixed set of initial conditions $\mathbf{r}_{i}(t=0)=\mathbf{r}_{i 0}, \quad i=1, \ldots, n$, Eq. (5) has a unique solution.

We note that Eq. (5) is invariant under the transformation $t \rightarrow-t, \mathbf{e} \rightarrow-\mathbf{e}$, which means the reversal of time and the driving force. Consequently, it is also invariant under the transformation $t \rightarrow-t, \mathbf{r}_{i} \rightarrow-\mathbf{r}_{i}$ for $i=1,2,3$ at a fixed $\mathbf{e}$. The system is thus reversible in the sense that there is an involution (a transformation that composed with itself yields the identity) in phase space which reverses the direction of time [23]. In addition, the phase-space volume is conserved: $\sum_{i} \nabla_{i} \cdot \dot{\mathbf{r}}_{i}=0$, although there is dissipation in the system (it is not Hamiltonian). As a consequence of this volume preservation, however, the dynamics cannot have any attractor.

It is worth mentioning that this equation is similar in spirit to that describing the dynamics of ideal point vortices in a two-dimensional fluid $[24,25]$. They are also freely advected in the flow field induced by the others. The vortices do not have self-velocities $\left(\mathbf{v}_{0}=\mathbf{e}=0\right)$, and the function $U(\mathbf{r})$ is then proportional to $1 / r$ according to the reciprocal distance dependence of the velocity field around a single vortex [24].

The relative motions of the particles are unaffected by the constant term $\mathbf{v}_{\mathbf{0}}$ or $\mathbf{e}$. For this motion, an alteration of length scale in the configuration is equivalent to a change in the time scale, since the remaining terms in Eq. (5) are homogeneous functions of the positions. Without any loss of generality, any convenient length in the initial configuration can be chosen as a unit [20].

\section{THREE PARTICLES IN A PLANE}

The analytical solution of Eq. (5) for $n=2$ (the twoparticle problem) is not difficult [1]. It corresponds to a parallel displacement of the pair in a direction being, in general, different from that of the driving force e. The first nontrivial case is the motion of three particles. Although the trajectories from a general initial configuration can be tracked only numerically, useful exact results are known for special configurations. Already Hocking recognized a conservation law [20]: The horizontal projection (more generally, a projection along e) of the triangle formed by the three particles is of constant area. Thus an initial configuration of zero projected area leads to a motion in a vertical plane. Periodic solutions belonging to symmetric initial configurations of nonzero projected area have been discussed by several authors $[20,22,26]$.

The three-particle problem in a vertical plane has also attracted considerable interest $[20,21,6-8,10,15]$. Periodic orbits were not found. Typically, after some "mixing" phase, during which the particles totally change their relative positions compared to the initial configuration, a couple is formed and the third particle lags behind (see Fig. 1). (A couple moves faster than an isolated particle.) It is also 


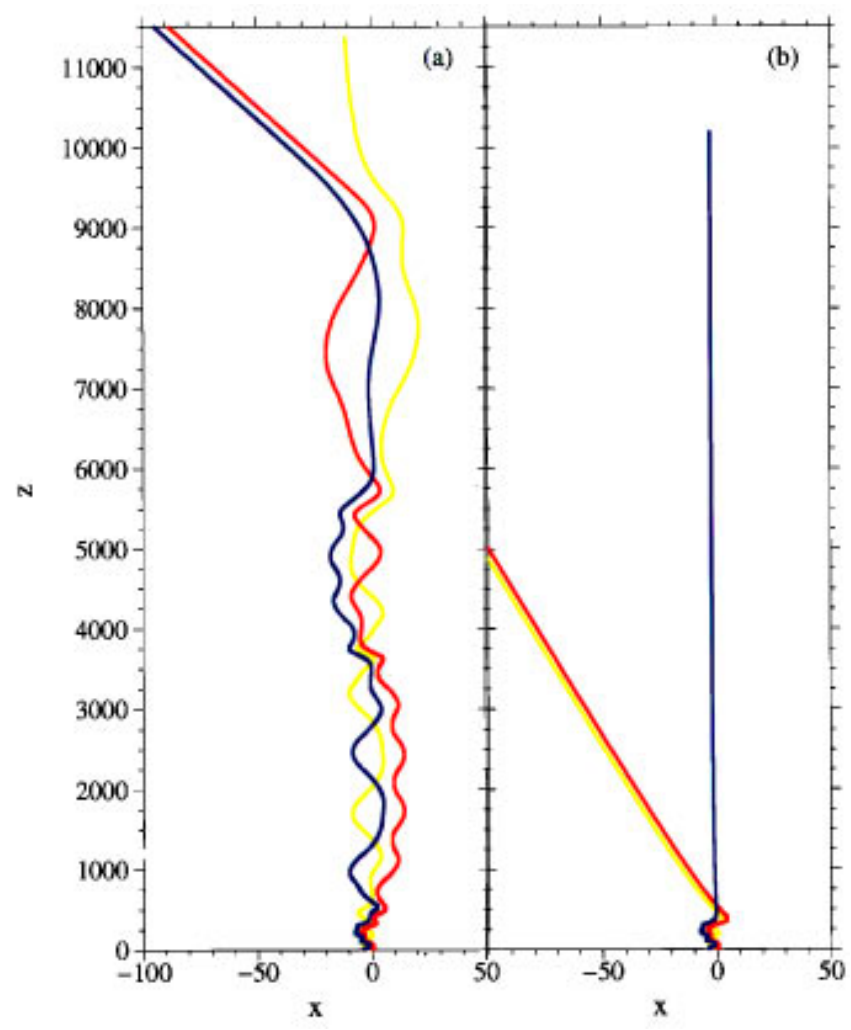

FIG. 1. (Color) Numerical solution of model (5) from two initial configurations for $10^{5}$ Stokes times. The particles started from the same height $z=0$. The horizontal locations $x$ were (a) $(-3.9050,-1.0,1.0)$, and (b) $(-3.9048,-1.0,1.0)$. The color coding for both cases is (blue, yellow, red). Note that the particle left behind and the length of the mixing phase are very different.

known from experiments [21] and simulations [20,7,8] that the final configuration depends very sensitively on the initial one.

We have systematically studied the Stokeslet model for three particles in a vertical plane. By introducing relative coordinates $\mathbf{r}_{12}=\mathbf{r}_{2}-\mathbf{r}_{1}$ and $\mathbf{r}_{23}=\mathbf{r}_{3}-\mathbf{r}_{2}$, we find for the relative motion

$$
\begin{aligned}
& \dot{\mathbf{r}}_{12}=\mathbf{U}\left(\mathbf{r}_{23}\right)-\mathbf{U}\left(\mathbf{r}_{12}+\mathbf{r}_{23}\right), \\
& \dot{\mathbf{r}}_{23}=\mathbf{U}\left(\mathbf{r}_{12}+\mathbf{r}_{23}\right)-\mathbf{U}\left(\mathbf{r}_{12}\right) .
\end{aligned}
$$

Here we have utilized that $\mathbf{U}(\mathbf{r})$ is an even function. Note that after the restriction to two-component vector variables, Eq. (6) still preserves the phase-space volume. In spite of this and the aforementioned reversibility property, Eq. (6) cannot be written in a Hamiltonian form [27]. In fact, the system seems to have no global conserved quantities. We solved these equations with a step-adaptive fourth order RungeKutta algorithm [28]. The simplicity of Eq. (6) allowed us to use a much higher resolution than in previous experimental or numerical studies.

The phase space of this system of equations is four dimensional. In order to gain insight into the complex geometrical structures underlying the dynamics, we fix one of the relative coordinates $\mathbf{r}_{12}$, at time zero, and monitor the changes due to varying the other initial coordinate $\mathbf{r}_{23}$. The $(x, z)$ plane defined by the two components of $\mathbf{r}_{23}$ will be

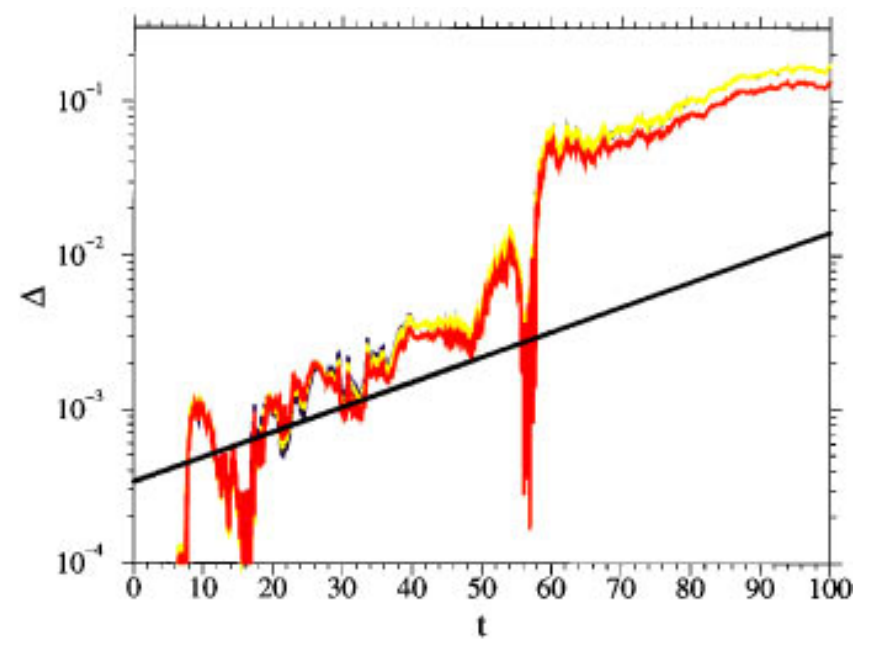

FIG. 2. (Color) The growth of the Euclidean distance $\Delta$ between unperturbed and perturbed trajectories as a function of time $t$. The blue, yellow, and red particles started from the same height $z=0$ at the horizontal locations $x=-2.9510,-1.0$, and 1.0, respectively. The initial perturbation imposed on the blue particle was $\delta x=10^{-10}$. The exponential fit (black) has a slope of 0.038 \pm 0.001 for each of the particles.

called the initial condition space, or the phase space, of the test particle (at fixed inital condition for the other two).

\section{RESULTS}

We first show the trajectories obtained by two simulations with slightly different initial conditions (see Fig. 1). The ultimate separation into a couple and an isolated particle is clear in both cases. A tiny change in the relative positions results, however, in a strong rearrangement. The mixing phases, during which all particles stay close to each other, have drastically different durations. Note, furthermore, that a completely different particle is left behind in these two cases.

We are now in a position to quantitatively characterize this sensitivity to initial conditions. A perturbation $\delta x(t=0)=10^{-10}$ was imposed on only one of the particles. (Note that a perturbation on a single particle changes all the trajectories as a consequence of strong hydrodynamical interaction.) We determined the Euclidean distance $\Delta=\sqrt{\left(x_{p}-x_{u}\right)^{2}+\left(z_{p}-z_{u}\right)^{2}}$ for each of the three particles, where the subscripts $p$ and $u$ belong to the perturbed and unperturbed positions at the same time. Figure 2 shows that the divergence has an overall exponential time dependence $\Delta(t) \sim \exp (\lambda t)$, and the fit gives an estimate for the first (local) Lyapunov exponent $\lambda=0.038 \pm 0.001$. Because of the reversibility property and the continuous time dependence, Eq. (6) must have three other (local) Lyapunov epxonents. Two of these vanish, and the last one is $-\lambda$ [29]. Note that other values of the first local Lyapunov exponent were obtained in the range between 0.0 (power-law divergence) and 0.1 depending on the initial configurations, but we found values around 0.04 to be representative. The positivity of the first Lyapunov exponent is a clear indication of chaos. In fact, this kind of chaos is of transient type [30], and has a finite average lifetime.

This situation is rather similar to what is called chaotic 


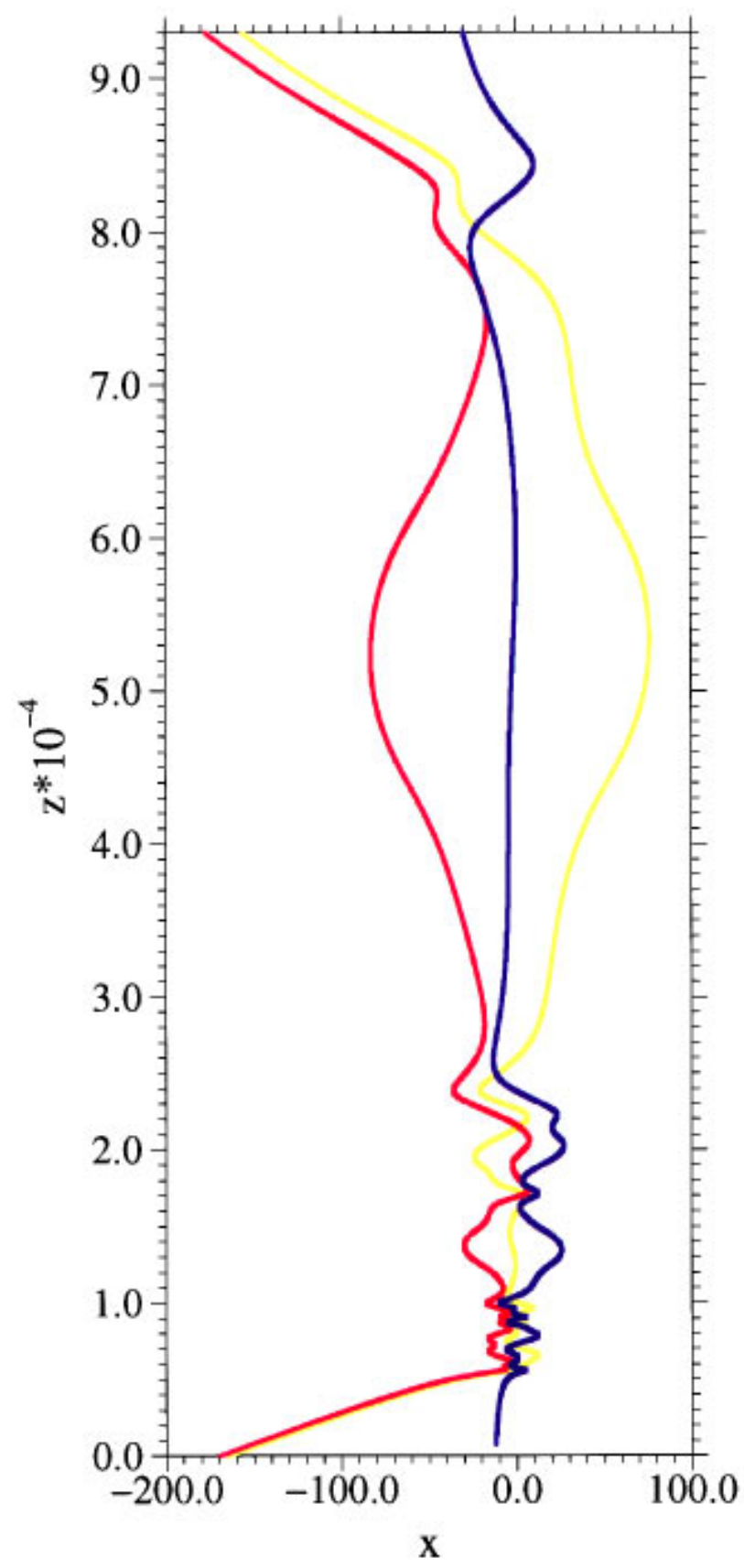

FIG. 3. (Color) Numerical solution of model (5) for $10^{6}$ Stokes times illustrating a scatteringlike process. The particles started from the following $(x, z)$ initial positions: Red $(-169.697802,0.0)$, yellow (-164.658 129, 0.973979$)$, and blue $(-12.157925$, 694.086 9269). Note that the couple formed after the scattering event need not coincide with the initial one. In this respect the example shown is special.

scattering [31]. The latter occurs in Hamiltonian systems characterized by a complicated interaction at short distances but without any interaction at asymptotically large distances. Examples are the motion of a single particle in a nontrivial potential of finite extension or classical models of chemical reactions [32]. Incoming particles then undergo a free motion, later they enter the region of strong interaction and, depending on their initital conditions, spend a longer or shorter time in this region. Ultimately all the particles escape to infinity in a free motion. The initial conditions used in the
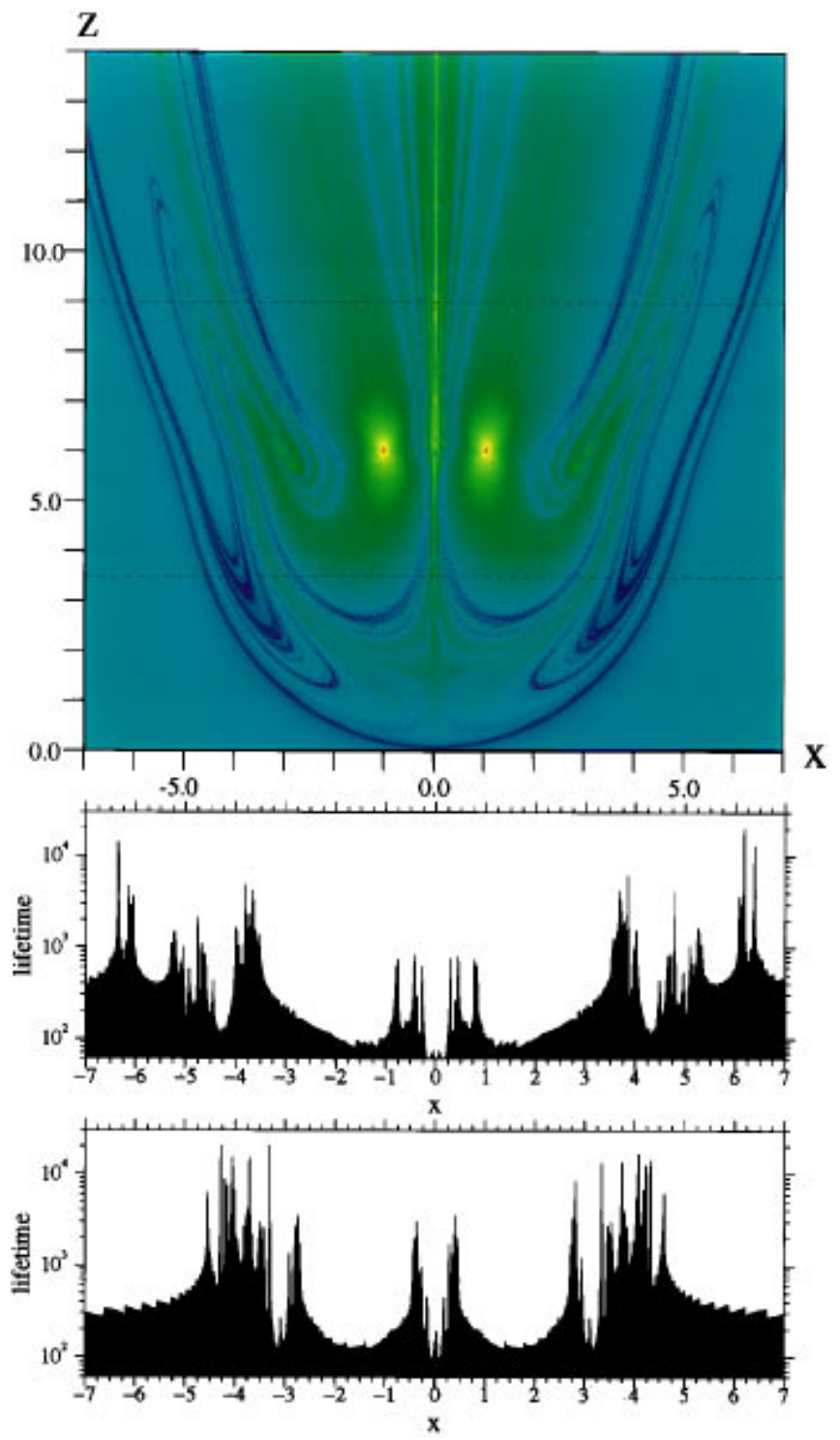

FIG. 4. (Color) Escape-time distribution from 640000 initial $(x, z)$ configurations with $\mathbf{r}_{12}=(2,0)$. The color scale from red across yellow, green, and blue to violet indicates increasing times from 0 to 10000 Stokes units. Two cross sections are explicitly shown at the heights of $z=9.0$ (middle) and $z=3.5$ (bottom), indicated by dotted lines.

Stokeslet model above correspond to placing the particles into the interaction region. We can, however, easily find other initial conditions where the motion starts with a phase of approach. An example is shown in Fig. 3. The analogy with a scattering process is clear: The asymptotic state and the interaction region correspond to configurations when at least one particle is far away from the others, and when all three particles are close to each other, respectively. In fact, the stay in the mixing phase is the analog, in the language of chemical reactions, of the creation of an intermediate complex of finite lifetime.

The lifetime distribution, i.e., the duration of the stay in the interaction region is known to be a rather irregular function in chaotic scattering. This irregularity is due to the existence of an underlying invariant chaotic saddle [30], which is a globally nonattracting set, and cannot be reached exactly. 
Z

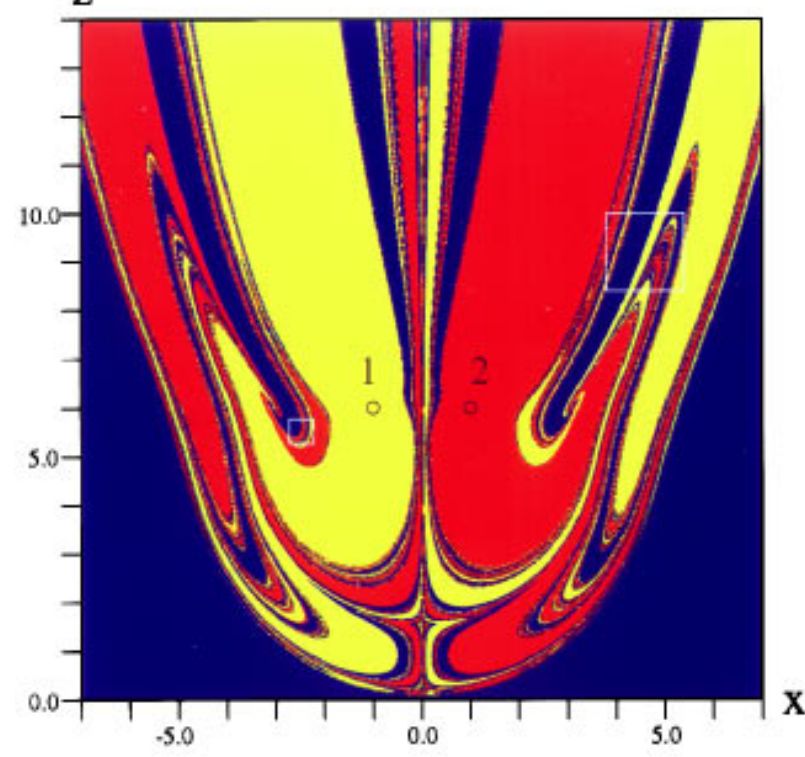

Z

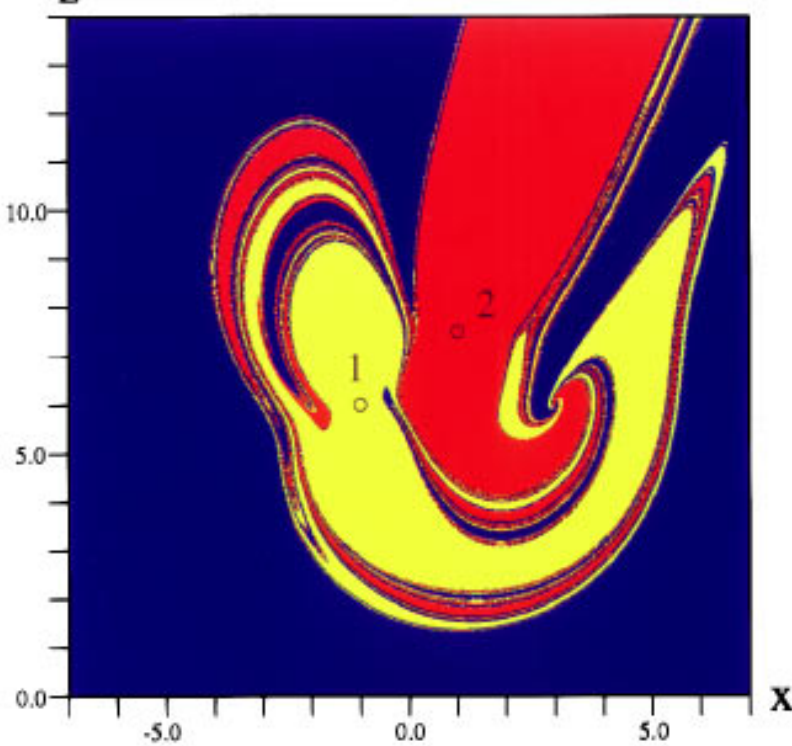

Z

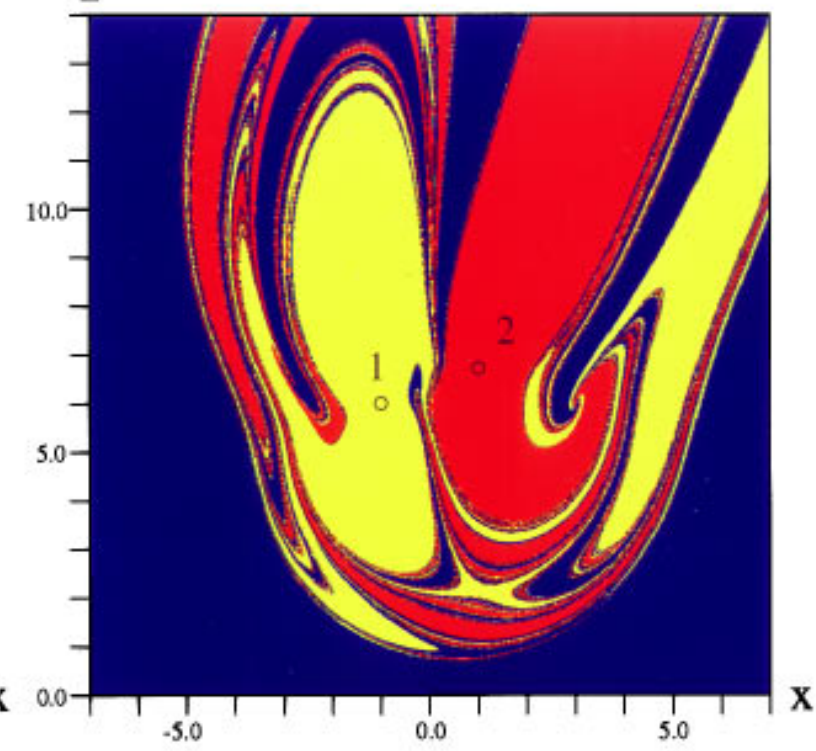

Z

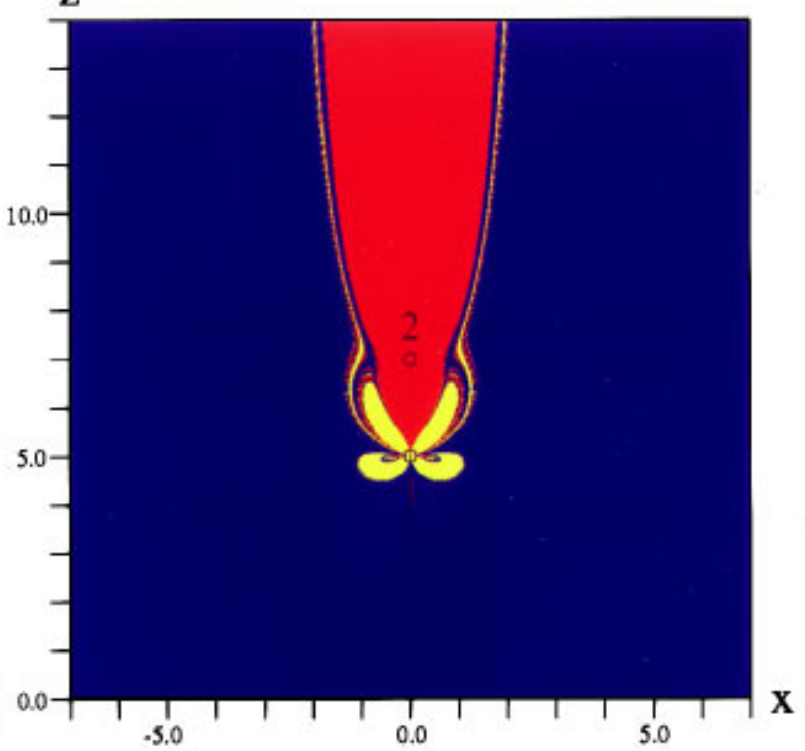

FIG. 5. (Color) Four sections of the initial condition space of the test particle. Particles 1 and 2 start always from the same $[x(t=0), z(t=0)]$ positions. (a) Top left, $(-1,6)$ and $(1,6)$; (b) top right, $(-1,6)$ and $(1,6.7)$; (c) bottom left $(-1,6)$ and $(1,7.5)$; $(\mathrm{d})$ bottom right, $(0,-5)$ and $(0,7)$, respectively. The final configurations from 640000 initial points are indicated in each figure. Different colors denote which particle is left behind. Red, particle 1; yellow, particle 2; blue, test particle. The thin white squares on the upper left panel show the regions zoomed with higher resolutions in Fig. 6.

Particles, however, can come arbitrarily close to it and exhibit chaotic motion before escaping. The chaotic saddle itself is a fractal with a local structure resembling the direct product of two Cantor sets in certain regions. It contains an infinite number of bounded orbits. Furthermore, there exists a complicated fractal curve, the so-called stable manifold, along which particles can hit the saddle (although, as mentioned above, the probability of falling exactly onto this curve is zero, because it is a fractal). Points where the lifetime distribution takes on infinitely large values correspond to initial conditions falling exactly on the stable manifold. Numerically very large values can really be obtained belonging to trajectories starting close to the stable manifold, and staying therefore a long time around the saddle.

In order to measure a lifetime distribution in the system of three particles, we say that an "escape" of a couple is established, if its vertical distance from the third particle left behind exceeds a threshold $z_{c}$. We found that at separations $z_{c} \geqslant 100.0$ the particle which is left behind has a negligible interaction with the pair, it moves vertically upwards with its own self-velocity. The lifetime or escape time $T$ depends sensitively on the initial configuration. To illustrate this, we fixed the initial value of $\mathbf{r}_{12}$ to be $(2,0)$ by letting particles 1 and 2 start in $(-1,6)$ and $(1,6)$, respectively, and simulated several trajectories with initial $\mathbf{r}_{23}=(x, z)$ values in a broad range. The lower part of Fig. 4 shows the dependence of the escape time on the test particle's initial $x$ coordinate at two fixed initial heights $z$. The distributions are rather irregular with regions of very wild changes over several orders of magnitude in the escape time $T$. The color plate was obtained 


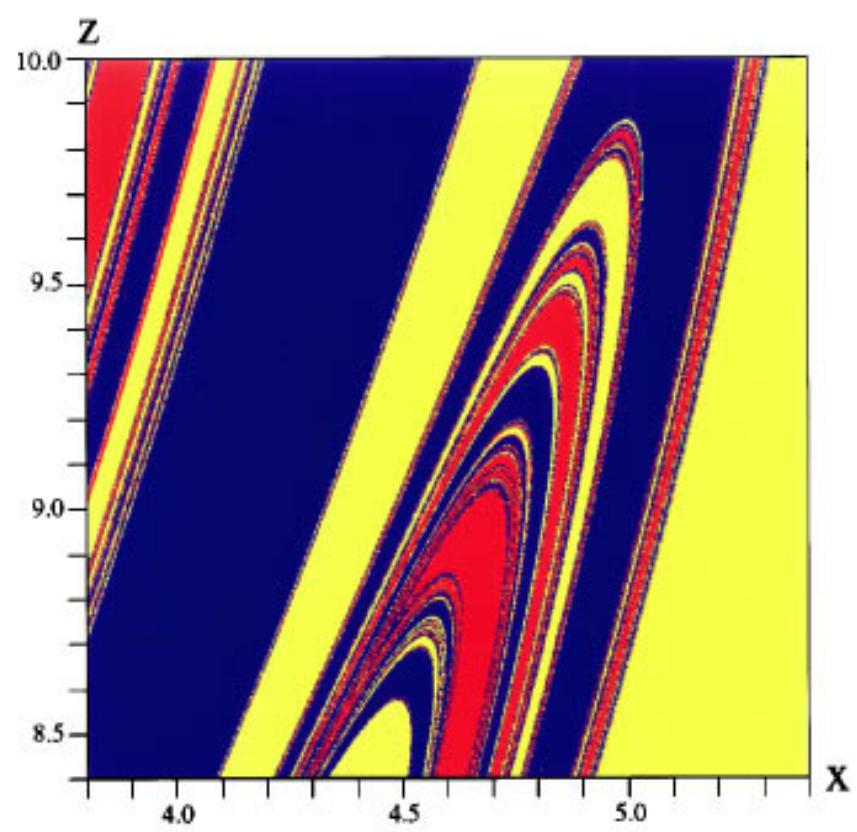

Z

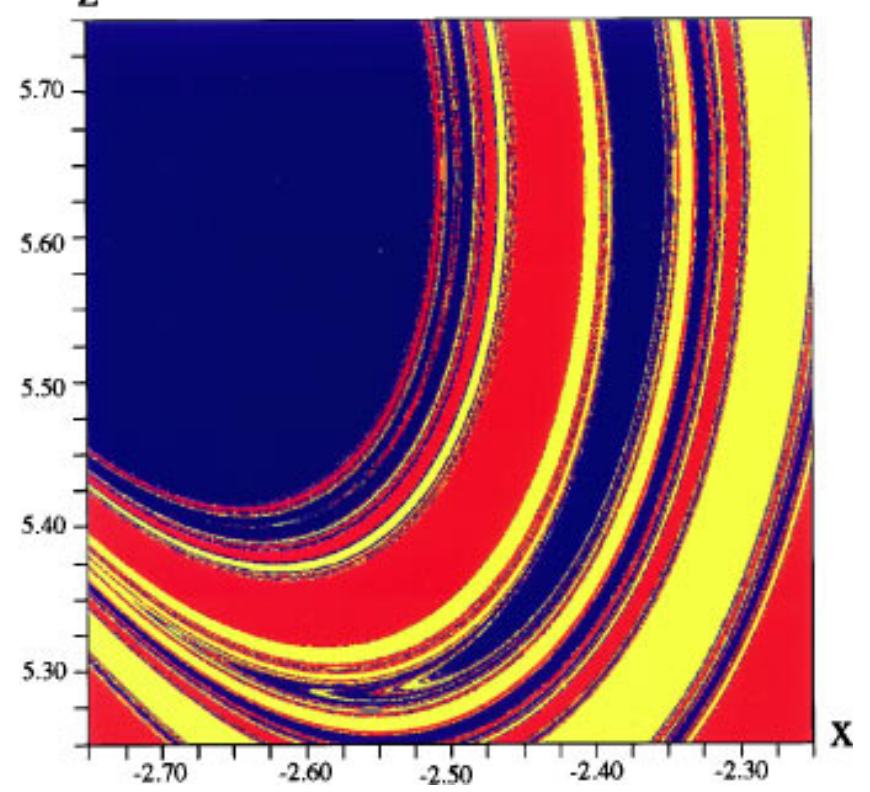

FIG. 6. (Color) Enlarged regions of the phase-space section shown in Fig. 5(a). The resolution is proportionally increased, 640000 initial configurations are plotted. The color coding is identical to that of Fig. 5.

by distributing initial points on the rectangle $|x| \leqslant 7$, $0<z<14$ uniformly. The color coding corresponds to the value of the escape time. Note that the escape time is very short close to the other two particles [i.e., around the points $(-1,6)$ and $(1,6)]$, because the velocity field diverges in the vicinity of any particle. Points with escape time on the order of 10000 Stokes units (violet) trace out with very good accuracy the chaotic saddle's stable manifold. The complicated winding and the fractal character of this curve is clear.

The coordinates of the test particle were chosen from an $(800 \times 800)$ grid with one of the grid points slightly displaced by $\delta x=\delta z=3 \times 10^{-7}$ from the fixed initial position
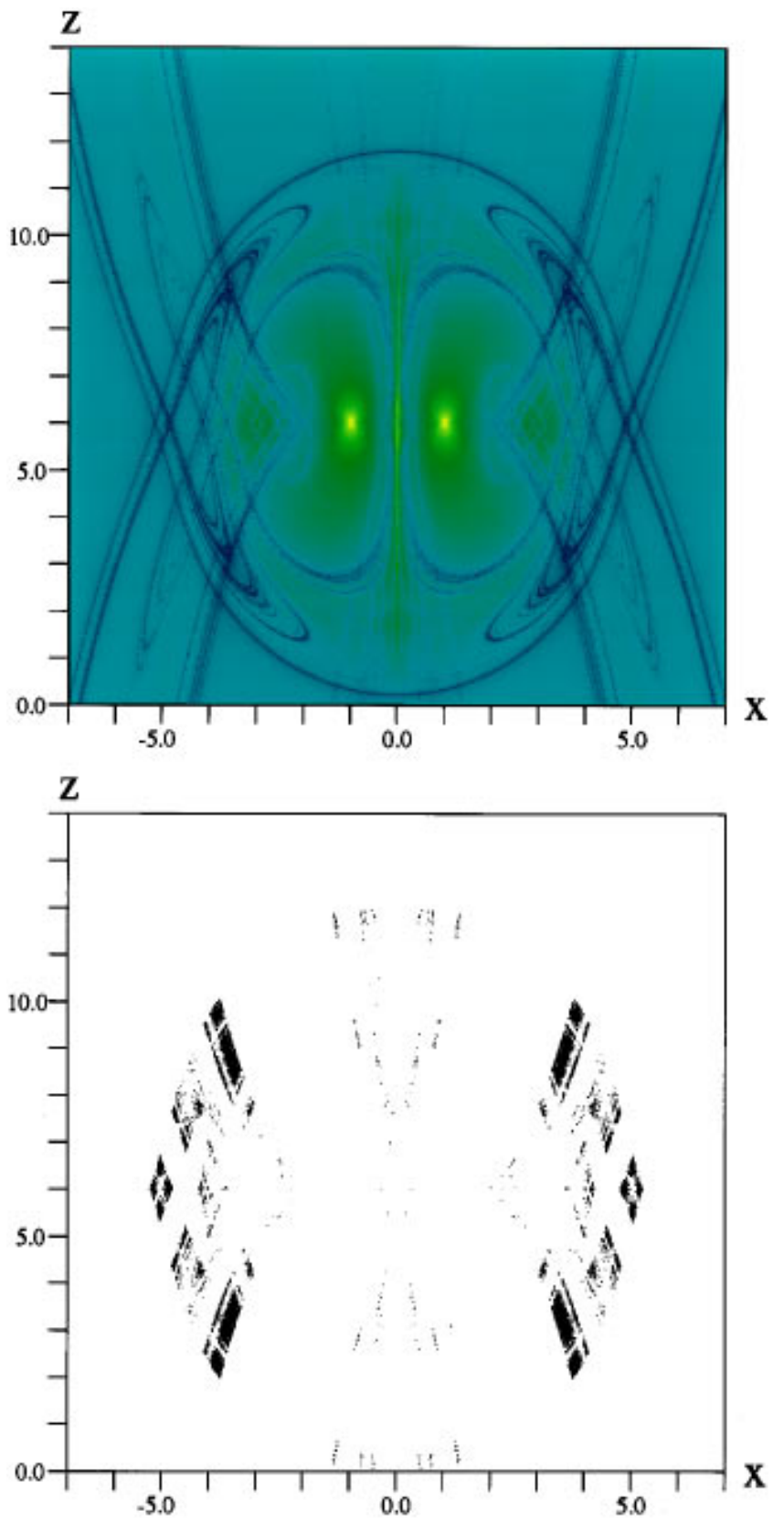

FIG. 7. (Color) Top: escape-time distribution in the forward and backward dynamics on the $(x, z)$ plane for $\mathbf{r}_{12}=(2,0)$. (The backward dynamics was evaluated explicitly on the original grid, it is not a mirror image. Hence the agreement with the reflected pattern is a direct assessment of the quality of the resolution.) The color scale from red across yellow, green, and blue to violet indicates increasing time from 0 to 20000 Stokes units (cf. Fig. 4). Bottom: the chaotic saddle on the $(x, z)$ plane, i.e., initial configurations belonging to long triplet orbits in both temporal directions are indicated by the black dots.

of one of the other two particles. This is because exactly symmetric initial configurations result in particle "collisions," which show up as divergences in the numerical integration process.

To obtain a further impression about the intricate phasespace structures, we consider now the same part of the test 
particle's initial condition space as in Fig. 4, but with a different coloring. The colors now mean different escape configurations: They correspond to the particle left behind in the asymptotic state. Figure 5(a) shows the results, Fig. 6 is magnification of the regions denoted by white squares in Fig. 5(a). It is natural that if the test particle is close to any of the other two, they form a couple and leave behind the particle that was originally furthest away from them. Thus, big unicolored regions appear around the points $(-1,6)$ and $(1,6)$ denoted by 1 and 2, respectively. It is less trivial that compact regions of a given color can be found also further away. The most surprising fact is, however, that they are separated by regions where different colors can come arbitrarily close to each other. A comparison with Fig. 4 shows that this takes place along the lines where the escape time is particularly large, i.e., along the stable manifold. In fact this manifold has an interesting topological property (just as in other scattering systems with at least three different exit modes [33]): Any neighborhood of any point on the manifold contains all three colors (cf. Figs. 5, 6). This shows that there is a strong mixing of the escape modes along the stable manifold which is another consequence of the sensitivity to initial conditions.

The dependence of the test particle's phase-space structures on the initial position of particles 1 and 2 is illustrated by Figs. 5(b)-5(d). Here a sequence of $\mathbf{r}_{12}$ values were taken corresponding to initial alignments deviating more and more from a horizontal one, and ending in a vertical arrangement. The figure indicates that a rotation of the initial vector $\mathbf{r}_{12}$ leads to a smooth deformation of the phase-space patterns.

It is instructive to construct the chaotic saddle itself, or at least a part of it. Since the saddle contains all the points that never escape either forward or backward in time, it can be obtained as the intersection of the stable manifolds of the direct and time inverted dynamics. Due to the reversibility property of Eq. (6) mentioned above, the latter is just the mirror image of the former with respect to the axis $z=6$ [34]. In other words, we obtain a rather accurate picture of the saddle by plotting points with very large escape times in both types of dynamics. Figure 7 shows the escape-time distribution of both the forward and backward dynamics on the $(x, z)$ plane with color coding. Points that belong simultaneously to large values in both dynamics are plotted in black in the lower part of Fig. 7 and correspond to points of the chaotic saddle in the initial condition space of the test particle. Note the striking direct product structure.

Besides the typical asymptotic states formed by a couple separated from a single particle, the system has more exceptional asymptotic states in which all three particles are far away from each other and move with their own selfvelocities upward. In such three-singlet states $\dot{\mathbf{r}}_{12}=\dot{\mathbf{r}}_{23}=0$. Our numerical procedure with a long duration of integration and a large fixed $z_{c}$ provides us with initial points that come after a long time close to a three-singlet state in both the forward and backward dynamics. In other words, the black points $\mathbf{r}_{23}$ of Fig. 7 along with $\mathbf{r}_{12}=(2,0)$ correspond to mid states of complicated trajectories which can be reached after an infinitely long time from some initial three-singlet configuration and which decay towards another three-singlet configuration. The property that the invariant manifolds of some asymptotic state provide a fractal foliation of the phase space (or initial condition space) has also been observed in


FIG. 8. (a) Result of the box counting for the chaotic saddle shown in Fig. 7. The size $\epsilon$ of the square boxes covering the image of the fractal set is measured in pixels, the resolution of the whole image was $800 \times 800$ pixels. The slope of the power-law fit is $-1.24 \pm 0.04$. (b) Normalized escape-time statistics $P(T)$ for the forward dynamics in the $(x, z)$ phase-space segment shown in Fig. 7. The slope of the exponential fit is $-0.013 \pm 0.002$.

three-degree-of-freedom scattering systems with energy conservation [36]. It is generally believed that a full chaotic saddle contains an infinite number of unstable periodic orbits, too [30]. This would imply that, in contrast to previous statements, the three-particle dynamics has unstable periodic solution as well, although they might be rather unstable and of rather long period [cf. trajectories at around $800<z<3000$ in Fig. 1(a)].

As a next step, one can determine some quantitative characteristics of the saddle [35]. Figures 4 and 5 suggest that the stable manifold in the four-dimensional phase space has a fractal dimension $3+D_{p}$ with $0<D_{p}<1$. Consequently, on the initial condition plane it appears as a curve of dimension $1+D_{p}$. Due to the reversibility property, the intersection of the stable and unstable manifolds in the full space is $2+2 D_{p}$ dimensional. The chaotic saddle on Fig. 7 should thus have dimension $2 D_{p}$. One of the simplest methods for measuring the dimension of a fractal object is the box counting: The number of two-dimensional boxes $N(\epsilon)$ covering the saddle as a function of box size $\epsilon$ scales with a nontrivial exponent $D_{\text {box }}$, see Fig. 8(a). The power-law fit gives an estimation $D_{b o x} \approx 1.2$. Consequently, $D_{p} \approx 0.6$ is the partial dimension of the saddle along the stable (unstable) manifold on the $(x, z)$ plane of Fig. 7.

Figure 8(b) shows the escape-time statistics $P(T)$, the probability density for finding an escape time $T$ in a large ensemble of particles. In the time regime $150<T<350$ it obeys an exponential decay $P(T) \sim \exp (-\kappa T)$ with a decay 
rate $\kappa \approx 0.013$. This is an important fingerprint of transient chaos too [30]. There is a fundamental relationship between the partial dimension $D_{p}$, the decay rate $\kappa$ and the first average Lyapunov exponent $\lambda$ [30]: $D_{p} \approx 1-\kappa / \lambda$. Our estimated parameters are consistent with this relationship in the given numerical accuracy.

The sharp jump in Fig. 8(b) at around $T \approx 350$ Stokes time is most probably associated with characteristic shapes in the trajectories. In the mixing phase, the number of "knots," where the three particles move close to each other, has an integer value. Starting from the initial conditions in the phase-space segment shown in Fig. 5(a), we find that the jump in $P(T)$ belongs to trajectories with three knots. Orbits with three knots have escape times dominantly around $T=350$. This behavior is characteristic only in the large, single-colored regions in the phase space where the trajectories are not sensitive strongly to small changes in the initial configurations. At smaller escape times most of the orbits have only two knots. Interestingly, neither the single knot orbits (there are not too many), nor orbits with a larger knot number have such a characteristic escape time. (Note that there is no direct relationship between the escape time and a characteristic "meeting time" at the last "knot," because the escape time contains an interval during which the couple moves away from the third particle to the critical distance $\left.z_{c}=100.\right)$

\section{DISCUSSION}

We pointed out in Sec. IV that the dynamics of three particles is chaotic, and it is associated with an invariant chaotic saddle. Since the Stokeslet model of point particles we used is the simplest approximation for describing hydrodynamical interactions in the zero Reynolds number limit, we should discuss to which extent our results can be valid at small but finite Reynolds numbers.

First of all, we compare the numerical results with related experiments. Jayaweera, Mason, and Slack [21] performed the first sedimentation experiment with three particles starting from a line. Later, Ganatos, Pfeffer, and Weinbaum [7] developed a numerical method to calculate particle velocities and drag coefficients for systems of identical spheres. They obtained a general agreement between the experimental and numerical results, however they noted some differences and pointed out again the sensitivity to initial configurations. We show a comparison between the experiments and our results in Fig. 9. We cannot expect a better agreement, especially due to the differences in the resolutions. In addition, the experiments were performed with finite spheres, where rotation, neglected in our treatment, can modify the trajectories.

As for corrections to the lowest order approximation, we have checked the effect of $O\left(1 / r^{3}\right)$ terms. The simplest Stokeslet velocity field Eq. (3) can be replaced in Eq. (4) by the full solution [4]

$$
\mathbf{u}^{\prime}(\mathbf{r})=\frac{3 a v_{0}}{4}\left(\frac{\mathbf{e}}{r}+\frac{(\mathbf{e} \cdot \mathbf{r}) \mathbf{r}}{r^{3}}+\frac{a^{2} \mathbf{e}}{3 r^{3}}-\frac{a^{2}(\mathbf{e} \cdot \mathbf{r}) \mathbf{r}}{r^{5}}\right)=a v_{0} \mathbf{U}^{\prime}(\mathbf{r})
$$

of the Stokes approximation fulfilling the no-slip boundary condition on the surface of the sphere. Using the proper units

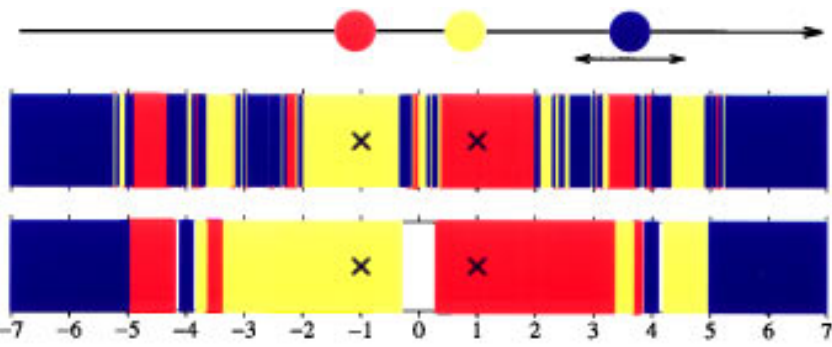

FIG. 9. (Color) Comparison of the simulations (top band) with the experimental results of Jayaweera, Mason, and Slack [21] (bottom band). Three particles sedimenting in a vertical plane from a horizontal line. The positions of the red and the yellow particles are fixed, indicated also by crosses in the bands. The blue test particle starts from different initial positions. Color stripes show which particle is left behind after a long time.

we recover the nondimensional forms (5) or (6) with $U$ replaced by $U^{\prime}$. Thus we can implement the same method for the numerical solutions. The result for the initial condition space of the test particle for $\mathbf{r}_{12}=(2,0)$ is plotted in Fig. 10 . A comparison with Fig. 5(a) shows that some new features appeared mostly around the fixed particles, but the overall phase-space structure remained considerably intact. One can also recognize a slight global shrinkage of the corresponding colored domains.

It is instructive to see the effect of different approximations considering special trajectories. Durlofsky, Brady, and Bossis [8] show the trajectories of three sedimenting spheres on a vertical plane starting from a line with a horizontal

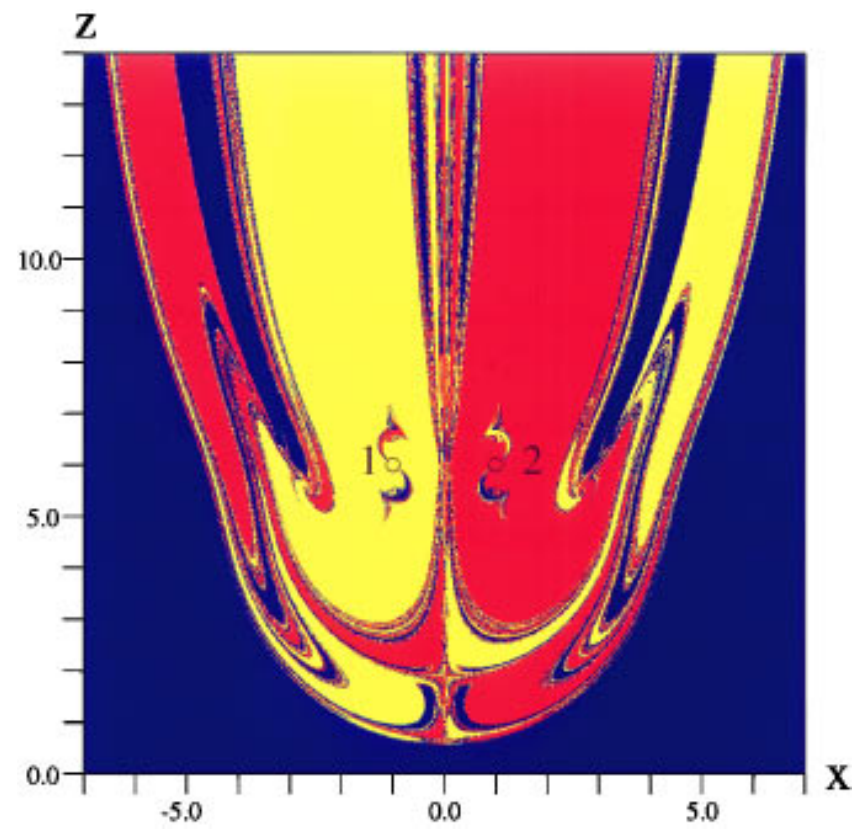

FIG. 10. (Color) Segment of the initial condition space $(x, z)$ for three particles of the modified velocity field Eq. (7) [cf. Fig. 5(a)]. Particles 1 and 2 start from the positions $(-1,6)$ and $(1,6)$, respectively, the third one is the test particle. The final configurations from 640000 initial points are indicated. The color coding is identical to that of Figs. 5 and 6. 


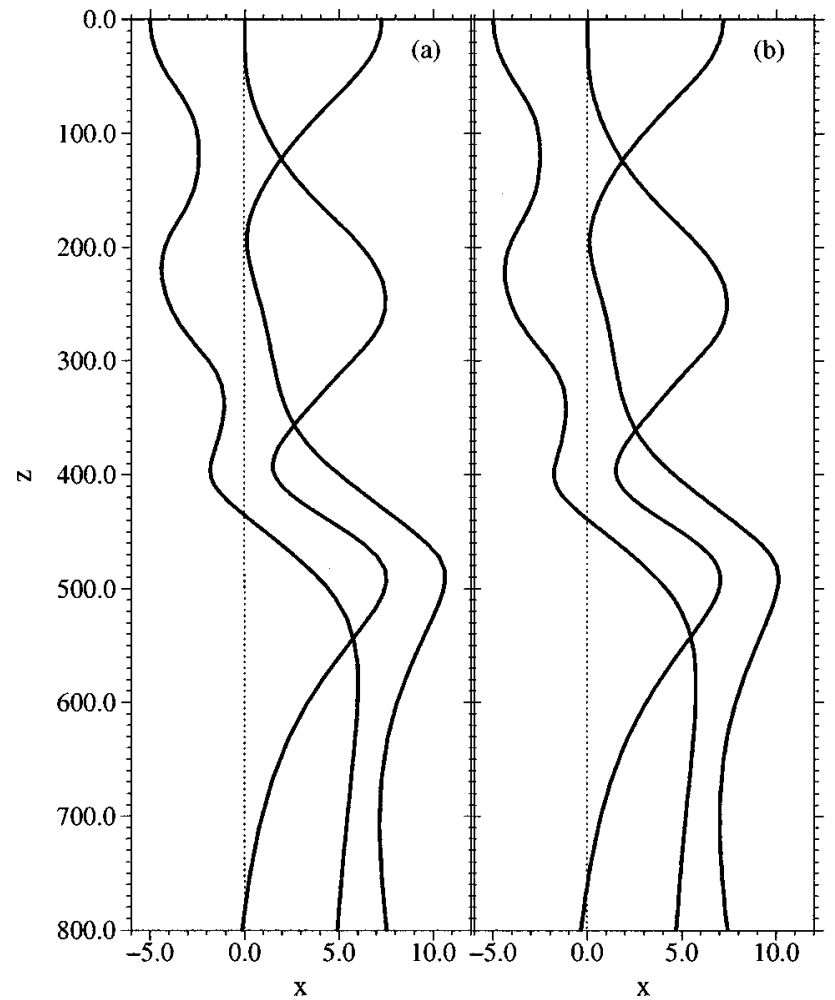

FIG. 11. Numerical solution of model (5) for two different velocity fields. The particles started from the same height $z=0$. The horizontal locations $x$ were (a) $(-5.0,0.0,7.258)$, velocity field (3); and (b) $(-5,0.0,7.165)$, velocity field (7). Axis $z$ is inverted.

spacing (-5,0,7) (see Ref. [8], Fig. 5(b) ). Their full hydrodynamical model also accounted for near-field lubrication effects and the dominant many-body interactions. The same initial configuration was used for benchmarking by Phillips [15], who implemented also an accurate model, and reproduced the trajectories almost identically (see Ref. [15], Fig. 2(a)). Our simple model (5) gives from the $(-5,0,7)$ initial configuration trajectories differing significantly from the cited ones. On the other hand, direct examination of many orbits gave the result that small changes in the initial configurations alter "smoothly" the resulting trajectories in the single-colored large regions in the phase space (Fig. 5). With a simple trial and error method we located the initial configuration $(-5,0,7.258)$ which reproduced the trajectories shown in Refs. [8] and [15], see Fig. 11(a). Based on the observation of the global shrinkage of the phase-space structures by introducing $O\left(1 / r^{3}\right)$ corrections, we found very quickly the proper initial configuration $(-5,0,7.156)$ for the modified field model (7) which also gives almost identical trajectories [Fig. 11(b)]. It is likely that the inclusion of additional terms, e.g., of those describing rotation, results in better and better approximations and modifies further the phase-space structures so that the initial configurations for the particular trajectories shown in Fig. 11 approach the "exact" ones for $(-5,0,7)$. Nevertheless, we believe that the simplest Stokeslet approximation with the leading $O(1 / r)$ terms captures already quite well the most essential features of the hydrodynamical interactions.

Here we would like to emphasize that the CPU time for obtaining the exact trajectories with full hydrodynamics is $\sim 12 \mathrm{~h}$ on a HP 715/100 workstation [37], while the Stokes-
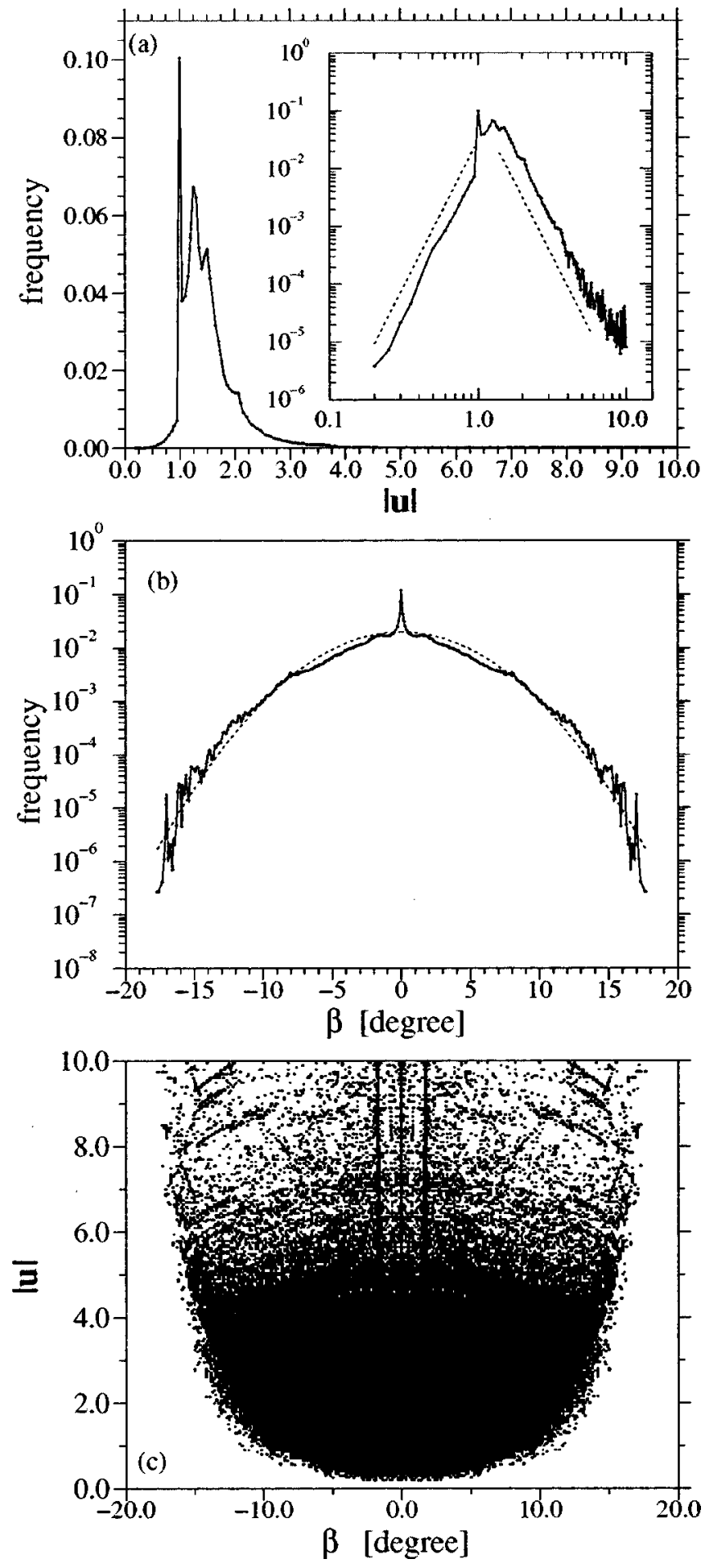

FIG. 12. (a) Empirical distribution of the magnitude $|\mathbf{u}|$ of the particles' instantaneous velocities during the full simulation of Eqs. (6) and (3) with initial conditions in the phase-space segment of Fig. 7. The inset shows the same distribution on a double logarithmic scale. The dotted lines are power-law fits for velocities both smaller and larger than the self-velocity $|\mathbf{u}|=1.0$, the exponents are close to +5 and -5 , respectively. (b) Distribution of the instantaneous moving directions $\beta$ with respect to the vertical axis (the particle left behind moves with $|\mathbf{u}|$ and $\beta=0)$. The dotted line shows a Gaussian fit $\exp \left(-0.03 \beta^{2}\right)$. (c) Correlation diagram between moving directions and velocity absolute values. The envelope of the set can be well fitted with an exponential function $|\mathbf{u}|_{\min }(|\beta|) \sim \exp (0.26|\beta|)$. 
let solution requires only $1.8 \times 10^{-6} \mathrm{~h}$ for a similar solution plotted in Fig. 11.

We mentioned in the Introduction the problem of swimming microorganisms. Although the hydrodynamical interaction between algae or bacteria is certainly weaker $[38,39]$ than that of, e.g., sedimenting particles, simply because they are driven by internal forces, these interactions may contribute to the collective behavior. For example, it is a common observation that "individual" trajectories tracked in an assembly of microswimmers show a rather noisy character (see, e.g., Fig. 2 in [39]). These trajectories can be characterized by rather wide distributions of swimming direction and velocity. In Fig. 12 we illustrate that the fully deterministic Stokeslet dynamics can also lead to rather wide and smooth distributions. Even more, the histogram of the moving directions [Fig. 12(b)] can be well approximated with a Gaussian, which is usually attributed to "pure" random noise. In our approach the stochastic behavior is due to a deterministic internal dynamics which is strongly chaotic. Unfortunately there is no well established simple far-field approximation for the velocity around a self-driven small "microswimmer," such as Eqs. (3) or (7), therefore we could not repeat the detailed analysis performed in the framework of the Stokeslet model.

In summary, we introduced the simplest Stokeslet model for studying the hydrodynamical interaction between microscopic particles driven by external forces. We illustrated the efficiency of the approximation by a detailed description of the three-particle motion in a vertical plane, and pointed out that the sensitivity to initial configurations is associated with a chaotic saddle resulting in a chaotic scattering like dynamics. Our resolution made it possible to locate the saddle and quantitatively characterize its dynamical and fractal properties. We argued that corrections do not alter the universal phase-space structures drastically, thus even the Stokeslet approximation should be able to capture the essential hydrodynamics in an interacting assembly.

This work was partially supported by NATO under Grant No. CRG960634 and the Hungarian National Science Foundation (OTKA) under Grant Nos. T17493, T19483, and F014967. Helpful discussions with A. Bringer, Z. Kovács, and L. Wiesenfeld are acknowledged.
[1] E.g., J. Happel and H. Brenner, Low Reynolds Number Hydrodynamics (Prentice Hall, Englewood Cliffs, NJ, 1965); S. Kim and S. J. Karrila, Microhydrodynamics (ButterworthHeinemann, Boston, 1991); Numerical Methods for the Simulation of Multi-Phase and Complex Flow, edited by T. M. Verheggen (Springer, New York, 1992); Numerical Methods in Multiphase Flows, edited by T. C. Crowe (American Society of Mechanical Engineers, New York, 1994).

[2] E.g., Y. Solomentsev and J. L. Anderson, J. Fluid Mech. 279, 197 (1994); P. Venema, ibid. 282, 45 (1995); M. Loewenberg and R. H. Davis, ibid. 288, 103 (1995).

[3] E.g., S. Childress, M. Levandowsky, and E. A. Spiegel, J. Fluid Mech. 63, 591 (1975); E. M. Purcell, Am. J. Phys. 45, 3 (1977); J. O. Kessler, Nature (London) 313, 218 (1985); J. O. Kessler, Contemp. Phys. 26, 147 (1985); T. J. Pedley and J. O. Kessler, Annu. Rev. Fluid Mech. 24, 313 (1992); J. O. Kessler and N. A. Hill, in Physics of Biological Systems: From Molecules to Species, edited by H. Flyvbjerg et al. (SpringerVerlag, Heidelberg, 1996).

[4] E. J. Hinch, in Disorder and Mixing, edited by E. Guyon et al. (Kluwer Academic, Dordrecht, 1988), p. 43.

[5] S. Kim and S. J. Karrila, Microhydrodynamics (ButterworthHeinemann, Boston, 1991).

[6] S. Leichtberg, S. Weinbaum, R. Pfeffer, and M. J. Gluckmann, Philos. Trans. R. Soc. London, Ser. A 282, 585 (1976).

[7] P. Ganatos, R. Pfeffer, and S. Weinbaum, J. Fluid Mech. 84, 79 (1978)

[8] L. Durlofsky, J. F. Brady, and G. Bossis, J. Fluid Mech. 180, 21 (1987).

[9] A. L. Fogelson and C. S. Peskin, J. Comput. Phys. 79, 50 (1988).

[10] M. T. Kamel and E. M. Tory, Powder Technol. 59, 227 (1989).

[11] H. J. H. Clercx and P. P. J. M. Schram, Physica A 174, 293 (1991); J. Chem. Phys. 96, 3137 (1992).

[12] A. J. C. Ladd, Phys. Fluids A 5, 299 (1993).
[13] D. L. Koch, Phys. Fluids 6, 2894 (1994).

[14] S. Schwarzer, Phys. Rev. E 52, 6461 (1995).

[15] R. J. Phillips, J. Fluid Mech. 315, 345 (1996).

[16] E. J. Hinch, in Disorder and Mixing, edited by E. Guyon et al.. (Kluwer Academic, Dordrecht, 1988), p. 153.

[17] A. J. C. Ladd, J. Fluid Mech. 271, 285 (1994).

[18] A. J. C. Ladd, J. Fluid Mech. 271, 1994.

[19] A. J. C. Ladd, Phys. Rev. Lett. 76, 1392 (1996).

[20] L. M. Hocking, J. Fluid Mech. 20, 129 (1964).

[21] K. O. L. F. Jayaweera, B. J. Mason, and G. W. Slack, J. Fluid Mech. 20, 121 (1964).

[22] R. E. Caflish, Ch. Lim, J. H. C. Luke, and A. S. Sangani, Phys. Fluids 31, 3175 (1988); M. Golubitsky, M. Krupa, and C. Lim, SIAM (Soc. Ind. Appl. Math.) J. Appl. Math. 51, 49 (1990); Ch. C. Lim, and I. H. McComb, J. Diff. Eqns. 121, 384 (1995).

[23] R. L. Devaney, Trans. Am. Math. Soc. 218, 89 (1976); J. A. G. Roberts, and G. R. W. Quispel, Phys. Rep. 216, 63 (1992).

[24] H. Aref, J. Fluid Mech. 143, 1 (1984); P. G. Saffman, Vortex Dynamics (Cambridge University Press, Cambridge, England, 1992).

[25] Á. Péntek, T. Tél, and Z. Toroczkai, Fractals 3, 33 (1995); Á. Péntek, Z. Toroczkai, T. Tél, C. Grebogi, and J. A. Yorke, Phys. Rev. E 51, 4076 (1995).

[26] M. T. Kamel and E. M. Tory, Powder Technol. 63, 187 (1990).

[27] The solutions to Eq. (6) possess a homogeneity property: rescaling all variables by the same factor $\Lambda: \mathbf{r} \rightarrow \Lambda \mathbf{r}$ is equivalent to rescaling the time by $\Lambda^{-2}: t \rightarrow \Lambda^{-2} t$.

[28] W. H. Press, S. A. Teukolsky, W. T. Vetterling, and B. P. Flannery, Numerical Recipes, 2nd ed. (Cambridge University Press, Cambridge, England, 1992).

[29] This follows from the fact that the homogeneity property (see [27]) defines a direction in the phase space (the set of geometrically similar configurations) along which no local divergence takes place. Thus, besides the translations along the trajectories, there is a second direction in which the local 
Lyapunov exponent is zero. Because of the conservation of phase-space volume, the sum of Lyapunov exponents must be zero and hence the last exponent must be $-\lambda$.

[30] T. Tél, in Directions in Chaos, edited by Hao Bai-Lin (World Scientific, Singapore, 1990), Vol. 3, pp. 149-221; in STATPHYS'19, edited by Hao Bai-lin (World Scientific, Singapore, 1996), pp. 346-362.

[31] E. Ott and T. Tél, CHAOS 3, 317 (1993); special issue on chaotic scattering, CHAOS 3(4) (1993).

[32] Z. Kovács and L. Wiesenfeld, Phys. Rev. E 51, 5476 (1995).

[33] L. Poon, J. Campos, E. Ott, and C. Grebogi, Int. J. Bifurcation Chaos Appl. Sci. Eng. 6, 251 (1996); Z. Toroczkai, G. Korolyi, A. Péntek, T. Tél, C. Grebogi, and J. Yorke, Physica A 239, 235 (1997).

[34] Due to the reversibility property of Eq. (6), it is true that the time reversed dynamics of a starting configuration $\mathbf{r}_{1}, \mathbf{r}_{2}, \mathbf{r}_{3}$ is the same as the direct dynamics of the mirror configuration $-\mathbf{r}_{1},-\mathbf{r}_{2},-\mathbf{r}_{3}$. Therefore, if a point $(x, z)$ in the relative coordinate representation [see Eq. (6)] is on the stable manifold of the chaotic saddle at a fixed $\mathbf{r}_{1}$ and $\mathbf{r}_{2}$, then the point $\left(-x,-z\right.$ ) belonging to $-\mathbf{r}_{1},-\mathbf{r}_{2}$ is on the unstable manifold. Thus, the unstable manifold on the $(x, z)$ plane is obtained, in general, by rotating the plot of the stable manifold by $180^{\circ}$ and interchanging the positions of particles 1 and 2. Due to the specific choice $z_{1}=z_{2}=6$ used in Figs. 4 and 5(a), the unstable manifold is just the mirror image of the stable one with respect to the axis $z=6$.

[35] I. M. Jánosi and T. Tél, Phys. Rev. E 49, 2756 (1994); I. M. Jánosi, L. Flepp, and T. Tél, Phys. Rev. Lett. 73, 529 (1994).

[36] S. Wiggins, Chaotic Transport in Dynamical Systems (Springer, New York, 1992); R. E. Gillilan and G. S. Ezra, J. Chem. Phys. 94, 2648 (1991); P. T. Boyd and S. L. W. McMillan, CHAOS 3, 507 (1993); Z. Kovács and L. Wiesenfeld (unpublished).

[37] R.J. Phillips (private communication).

[38] D. C. Guell, H. Brenner, R. B. Frankel, and H. Hartman, J. Theor. Biol. 135, 525 (1988); M. Ramia, D. L. Tullock, and N. Phan-Thien, Biophys. J. 65, 755 (1993).

[39] T. J. Pedley and J. O. Kessler, J. Fluid Mech. 212, 155 (1990). 